Bull. Austral. Math. Soc.

$49 \mathrm{~J} 40,49 \mathrm{~J} 27,47 \mathrm{H} 04$

VOL. 54 (1996) [473-481]

\title{
EXISTENCE THEOREMS FOR VECTOR VARIATIONAL INEQUALITIES
}

\author{
ARis Danillidis and Nicolas Hadjisavvas
}

\begin{abstract}
Given two real Banach spaces $X$ and $Y$, a closed convex subset $K$ in $X$, a cone with nonempty interior $C$ in $Y$ and a multivalued operator from $K$ to $2^{L(X, Y)}$, we prove theorems concerning the existence of solutions for the corresponding vector variational inequality problem, that is the existence of some $x_{0} \in K$ such that for every $x \in K$ we have $A\left(x-x_{0}\right) \notin-\operatorname{int} C$ for some $A \in T x_{0}$. These results correct previously published ones.
\end{abstract}

\section{INTRODUCTION}

Let $X, Y$ be real Banach spaces, $K$ be a closed, convex subset of $X$ and $L(X, Y)$ be the set of all continuous linear operators from $X$ to $Y$. Let further $T: K \rightarrow$ $2^{L(X, Y)} \backslash\{\emptyset\}$ be a multivalued operator and $C: K \rightarrow 2^{Y}$ be a multivalued mapping such that for each $x \in K, C(x)$ is a cone with nonempty interior int $C(x)$. The purpose of this paper is to study the existence of solutions for the vector variational inequality problem (VVIP):

$$
\exists x_{0} \in K: \forall x \in K, \exists A \in T x \text { such that } A\left(x-x_{0}\right) \notin-\operatorname{int} C\left(x_{0}\right) .
$$

In case $Y=\mathbf{R}, C(x)=\mathbf{R}^{+}$, the VVIP reduces to the well-known variational inequality problem [13]. The VVIP was introduced by Gianessi [8] for the case $Y=\mathbf{R}^{n}$ and was subsequently studied by many other authors $[2,3,4,14,17]$ in connection with vector optimisation. Theorems asserting the existence of solutions of the VVIP are contained in [3, Theorem 2.1] for single-valued, monotone operators $T$, where $Y$ has a constant cone $C$ (that is, not depending on $x$ ), in [2, Theorem 2.1] for $T$ a single-valued, monotone operator, where $Y$ is equipped with a non-constant $C(x)$ and in [14, Theorem 2.1] for multivalued, pseudomonotone operators $T$, with $C(x)$ constant. However, the proofs of all these theorems contain a mistake: a certain set defined in these papers in asserted to be weakly compact, while this is not the case (see Remark 2 at the end of the present paper for details).

In the following paragraph we prove the existence of a solution of the VVIP for a multi-valued, monotone operator [9] with constant cone $C$ (Theorem 3). We also prove

Received 4 January 1996

Copyright Clearance Centre, Inc. Serial-fee code: 0004-9729/96 \$A2.00+0.00. 
the existence of solutions for multivalued, pseudomonotone or quasimonotone operators with values consisting of completely continuous operators.

We now recall some definitions and fix our notation. A cone $C$ in $Y$ is a nonempty, convex, proper subset of $Y$, such that for all $\lambda \geqslant 0, y \in C$, we have $\lambda y \in C$. The dual cone $C^{*}$ of $C$ is the set of all $f$ in the dual space $Y^{*}$ such that $f(y) \geqslant 0$, for all $y \in C$.

If $C$ is closed, then

$$
y \in C \Leftrightarrow f(y) \geqslant 0, \text { for all } f \in C^{*} .
$$

On the other hand, if int $C \neq \emptyset$, then

$$
y \in \operatorname{int} C \Leftrightarrow f(y)>0 \text {, for all } f \in C^{*} \backslash\{0\} .
$$

Note that in both cases we have $C^{*} \neq\{0\}$. We refer the reader to [11] for these and other properties of cones.

Now let $C: K \rightarrow 2^{Y}$ be a multivalued mapping such that for each $x \in K, C(x)$ is a cone with nonempty interior. A multivalued operator $T: K \rightarrow 2^{L(X, Y)} \backslash\{\emptyset\}$ is called:

(i) monotone [9], if for all $x, y \in K$ and all $A \in T x, B \in T y$ we have $(B-A)(y-x) \in C(x)$.

(ii) (weakly) pseudomonotone [14], if for all $x, y \in K$ and $A \in T x$, $A(y-x) \notin-\operatorname{int} C(x)$ implies $B(y-x) \notin-\operatorname{int} C(x)$, for all (for some) $B \in T y$.

(iii) (weakly) quasimonotone, if for all $x, y \in K$ and $A \in T x, A(y-x) \notin$ $-C(x)$ implies $B(y-x) \notin-$ int $C(x)$, for all (for some) $B \in T y$.

It is obvious that (weak) quasimonotonicity is implied by (weak) pseudomonotonicity, which in turn, is implied by monotonicity. These notions generalise the well-known corresponding ones for the case $Y=\mathbf{R}[12,15]$.

The strong operator topology (SOT) on $L(X, Y)$ is the weakest topology for which the functions $L(X, Y) \ni A \rightarrow A x \in Y$ are continuous, for every $x \in X$. The multivalued operator $T$ is called upper hemicontinuous, if its restriction on line segments is SOT-upper semicontinuous. An operator $A \in L(X, Y)$ is called completely continuous, if it maps weakly convergent sequences to strongly convergent ones [5]. Any compact operator is completely continuous. The converse is not true, since the identity mapping in $\ell_{1}$ is completely continuous without being compact [6]. If $Y$ is finite-dimensional, all elements of $L(X, Y)$ are obviously completely continuous operators.

A point $x_{0} \in K$ is called an inner point $[10]$ or relative quasi-interior point $[1]$ of $K$, if for all $f \in X^{*}$, we have

$$
\forall x \in K, f\left(x-x_{0}\right) \geqslant 0 \Rightarrow \forall x \in K, f\left(x-x_{0}\right)=0 .
$$


In other words, $x_{0}$ is an inner point of $K$ if every closed hyperplane which supports $K$ at $x_{0}$, necessarily contains $K$.

The set of inner points of $K$ is denoted by inn $K$. Note that interior points of $K$ are also inner points, since in this case the above implication holds vacuously. In fact, whenever int $K \neq \emptyset$, it can be shown that int $K=\operatorname{inn} K$. However, for any separable $K$ we have inn $K \neq \emptyset$, even if $\operatorname{int} K=\emptyset[1,10]$. In $[1,10]$ it was also shown that inn $K$ is lineally full in $K$, that is for every $x \in$ inn $K$ and every $y \in K$, we have $\{t x+(1-t) y: t \in(0,1]\} \subseteq$ inn $K$.

For any $S \subseteq L(X, Y)$ and $x \in X, S(x)$ will denote the set $\{A x: A \in S\}$.

\section{The MAIN RESUlts}

In what follows, $X$ and $Y$ will be Banach spaces. Unless explicitly mentioned, we shall always consider the weak topology on $X$, the norm topology on $Y$ and the strong operator topology on $L(X, Y) . K$ will be a nonempty closed, convex subset of $X$ and $C: K \rightarrow 2^{Y}$ a multifunction, such that $C(x)$ is a cone with nonempty interior for each $x \in K$. We set $D(x)=Y \backslash(-\operatorname{int} C(x))$ and for any operator $T: K \rightarrow 2^{L(X, Y)} \backslash\{\emptyset\}$ we define the multifunctions:

$$
\begin{aligned}
& G(y)=\{x \in K: \exists A \in T x \text { such that } A(y-x) \in D(x)\} \\
& F(y)=\{x \in K: \exists B \in T y \text { such that } B(y-x) \in D(x)\}
\end{aligned}
$$

Let $S$ be the set of all $x \in K$ such that relation (1) holds, that is, $S$ is the solution set of the VVIP. We note that $S=\bigcap_{y \in K} G(y)$.

We begin with some lemmas:

Lemma 1. Let $K$ be (weakly) compact. Then $\bigcap_{y \in K} \overline{G(y)} \neq \emptyset$.

Proof: According to K. Fan's lemma [7], it is sufficient to show that for any $x=\sum_{i=1}^{n} \lambda_{i} x_{i}$, with $x_{i} \in G\left(x_{i}\right), \lambda_{i} \in[0,1], \sum_{i=1}^{n} \lambda_{i}=1$, we have $x \in \bigcup_{i=1}^{n} G\left(x_{i}\right)$. Indeed, were this not the case, we would have $x \notin G\left(x_{i}\right)$ for all $i$ 's, so for all $A \in T x$ we would have $A\left(x_{i}-x\right) \in-\operatorname{int} C(x)$. Since $-\operatorname{int} C(x)$ is convex, this would imply $0=\sum_{i=1}^{n} \lambda_{i} A\left(x_{i}-x\right) \in-\operatorname{int} C(x)$, a clear contradiction.

LEMma 2. Let $T$ be upper hemicontinuous. Then $\bigcap_{y \in K} F(y) \subseteq \bigcap_{y \in K} G(y)$. If, in addition, inn $K \neq \emptyset$ and $T$ has compact values, then $\bigcap_{y \in K} F(y)=\bigcap_{y \in \operatorname{inn} K} F(y)$.

Proof: Assume first that there exists $x \in \bigcap_{y \in K} F(y)$ such that $x \notin \bigcap_{y \in K} G(y)$. Then there would exist $y \in K$ such that $(T x)(y-x) \subseteq-\operatorname{int} C(x)$. Set $x_{t}=t y+$ 
$(1-t) x, t \in(0,1)$. Since $-\operatorname{int} C(x)$ is open and $T$ is upper hemicontinuous, there exists $\delta>0$ such that $\left(T x_{t}\right)(y-x) \subseteq-\operatorname{int} C(x)$, for all $t \in(0, \delta)$. Since $t(y-x)=$ $x_{t}-x$ and $-\operatorname{int} C(x)$ is a cone, we deduce that $\left(T x_{t}\right)\left(x_{t}-x\right) \subseteq-\operatorname{int} C(x)$, that is $x \notin F\left(x_{t}\right)$, a contradiction. This proves the inclusion.

Now suppose that inn $K \neq \emptyset$. Suppose that there exists $x \in \bigcap_{y \in \operatorname{inn} K} F(y)$ such that $x \notin \bigcap_{y \in K} F(y)$. Then for some $y \in K$, we would have

$$
(T y)(y-x) \subseteq-\operatorname{int} C(x)
$$

Since $(T y)(y-x)$ is compact by assumption, relation (6) implies that there exists $\varepsilon>0$ such that

$$
(T y)(y-x)+B_{\varepsilon}+B_{\varepsilon} \subseteq-\operatorname{int} C(x)
$$

where $B_{\varepsilon}=\{x \in X:\|x\| \leqslant \varepsilon\}$.

We choose $z \in$ inn $K$ and set $y_{t}=t z+(1-t) y, t \in(0,1]$. Since inn $K$ is lineally full, we have $y_{t} \in$ inn $K$, so $x \in F\left(y_{t}\right)$. We also have

$$
\left(T y_{t}\right)\left(y_{t}-x\right) \subseteq\left(T y_{t}\right)(y-x)+\left(T y_{t}\right)\left(y_{t}-y\right)
$$

Upper hemicontinuity shows that for $t$ sufficiently small, $\left(T y_{t}\right)(y-x) \subseteq(T y)(y-x)+$ $B_{\varepsilon}$. On the other hand, since $T$ has compact values and is upper hemicontinuous, the image of any line segment by $T$ is compact; hence, for small $t$ we have: $\left(T y_{t}\right)\left(y_{t}-y\right)=$ $t\left(T y_{t}\right)(z-y) \subseteq B_{\varepsilon}$. Hence, relations (7) and (8) imply $\left(T y_{t}\right)\left(y_{t}-x\right) \subseteq-\operatorname{int} C(x)$, that is, $x \notin F\left(y_{t}\right)$, a contradiction. This shows that $\bigcap_{y \in K} F(y)=\bigcap_{y \in \operatorname{inn} K} F(y)$.

LEMMA 3. Suppose that $K$ is compact and for some $y \in K, T(y)$ is norm compact and its elements are completely continuous operators. Suppose further that the graph of $D$ is sequentially closed in $X \times Y$. Then $F(y)$ is closed.

Proof: Let $x \in \overline{F(y)}$. By Eberlein's theorem, there exists a sequence $\left(x_{n}\right)_{n \in \mathbb{N}} \subset$ $F(y)$ converging to $x$. Then for any $n \in \mathrm{N}$, there exists $B_{n} \in T y$ such that $B_{n}\left(y-x_{n}\right) \in D\left(x_{n}\right)$. Since $T y$ is norm compact, we may assume with no loss of generality that $\left(B_{n}\right)_{n \in N}$ norm-converges to some $B \in T y$. Since $B$ is completely continuous, we have $B x_{n} \rightarrow B x$, so using a standard argument, we conclude that $B_{n}\left(y-x_{n}\right) \rightarrow B(y-x)$. The sequential closedness of the graph of $D$ implies that $B(y-x) \in D(x)$, that is $x \in F(y)$, so $F(y)$ is closed.

LEMMA 4. Suppose that $T$ is weakly quasimonotone and upper hemicontinuous, with compact values. Then for all $y \in \operatorname{inn} K$ we have $G(y) \subseteq F(y) \cup S$.

Proof: Let $x \in G(y)$ be such that $x \notin F(y)$. We shall show that $x \in S$. The assumption on $x$ implies that there exists $A \in T x$ such that $A(y-x) \notin-\operatorname{int} C(x)$. 
In addition, $A(y-x) \in-C(x)$, since otherwise the weak quasimonotonicity would imply that $x \in F(y)$. Hence $A(y-x)$ belongs to the boundary of $-C(x)$, so by the Hahn-Banach theorem there exists an $f \in Y^{*}$ such that $f(A(y-x)) \geqslant f(z)$, for all $z \in-C(x)$. Since $-C(x)$ is a cone containing $A(y-x)$, we easily deduce that

$$
(f \circ A)(y-x)=0 \geqslant f(z), \text { for all } z \in-C(x)
$$

so, in particular

$$
(f \circ A)(y)=(f \circ A)(x) .
$$

We now show that

$$
(f \circ A)(x)=(f \circ A)(y) \geqslant(f \circ A)(z), \quad \forall z \in K .
$$

Indeed, suppose to the contrary, that $(f \circ A)(z)>(f \circ A)(x)$ for some $z \in K$. Set $y_{t}=t z+(1-t) y, t \in(0,1)$. Obviously $(f \circ A)\left(y_{t}-x\right)>0$, for all $t \in(0,1)$, so (9) implies $A\left(y_{t}-x\right) \notin-C(x)$. Using the weak quasimonotonicity, we get

$$
\left(T y_{t}\right)\left(y_{t}-x\right) \cap D(x) \neq \emptyset .
$$

On the other hand, $x \notin F(y)$, which means that $(T y)(y-x) \subset-\operatorname{int} C(x)$. Using the same argument as in the second part of the proof of Lemma 2, we conclude that for $t$ sufficiently small we have $x \notin F\left(y_{t}\right)$, a contradiction.

Hence (10) holds. Since $y \in$ inn $K$, we deduce that $(f \circ A)(x)=(f \circ A)(y)=$ $(f \circ A)(z), \forall z \in K$; that is, $(f \circ A)(z-x)=0, \forall z \in K$. According to (9), $f$ belongs to the polar cone of $C(x)$, hence relation (3) implies $A(z-x) \notin-\operatorname{int} C(x)$, for all $z \in K$, that is, $x \in S$.

THEOREM 1. Suppose that $T$ is upper hemicontinuous and for all $y \in K, T(y)$ is norm compact and its elements are completely continuous operators. Let the graph of $D$ be sequentially closed in $X \times Y$ and $K$ be compact. Then in each of the following cases, the VVIP has a solution:

(a) $T$ is weakly pseudomonotone,

(B) $T$ is weakly quasimonotone and inn $K \neq \emptyset$.

Proof: (a). If $T$ is weakly pseudomonotone, then for all $y \in K$ we have: $G(y) \subseteq$ $F(y)$, so invoking Lemma 3 we get $\overline{G(y)} \subseteq F(y)$. Combining now Lemmas 1 and 2 we get

$$
\emptyset \neq \bigcap_{y \in K} \overline{G(y)} \subseteq \bigcap_{y \in K} F(y) \subseteq \bigcap_{y \in K} G(y)=S,
$$

hence $S$ is nonempty. 
$(\boldsymbol{\beta})$. Let $T$ be weakly quasimonotone. Suppose $S=\emptyset$. Then Lemmas 3 and 4 show that $\overline{G(y)} \subseteq F(y)$, for all $y \in$ inn $K$. Hence an application of Lemmas 1 and 2 gives

$$
\emptyset \neq \bigcap_{y \in K} \overline{G(y)} \subseteq \bigcap_{y \in \operatorname{inn} K} \overline{G(y)} \subseteq \bigcap_{y \in \operatorname{inn} K} F(y)=\bigcap_{y \in K} F(y) \subseteq \bigcap_{y \in K} G(y)=S,
$$

which is a contradiction. Thus $S \neq \emptyset$.

Theorem 2 replaces the hypothesis of (weak!) compactness of $K$ by a coercivity condition. We assume for simplicity that $X$ is reflexive.

THEOREM 2. Let $X$ be a reflexive Banach space. The conclusion of the Theorem 1 still holds if the assumption " $K$ is compact" is replaced by the following coercivity condition:

"There exists an $R>0$ such that for all $x \in K,\|x\| \geqslant R$, there exists a $z \in K$, $\|z\|<R$, such that $(T x)(z-x) \subseteq-C(x)$."

Proof: Define $K_{1}=\{x \in K:\|x\| \leqslant R\}$. Then $K_{1}$ is a nonempty, convex, compact subset of $X$.

We consider two cases:

(a) If $T$ is pseudomonotone, then by Theorem 1 the VVIP on $K_{1}$ has a solution $x_{0}$. By the coercivity condition, there exists a $z \in K,\|z\|<R$, such that

$$
\left(T x_{0}\right)\left(x_{0}-z\right) \subseteq C\left(x_{0}\right)
$$

(if $\left\|x_{0}\right\|<R$, we may take $z=x_{0}$ ). Now given $x \in K$, there exists $t \in(0,1)$ such that $x_{t}=t z+(1-t) x \in K_{1}$. By the definition of $x_{0}$, there exists $A \in T x_{0}$, such that $A\left(x_{t}-x_{0}\right) \notin-\operatorname{int} C\left(x_{0}\right)$. Combining the latter with (12), we easily deduce that $t A\left(x_{0}-z\right)+A\left(x_{t}-x_{0}\right) \notin$ $-\operatorname{int} C\left(x_{0}\right)$, that is, $A\left(x-x_{0}\right) \notin-\operatorname{int} C\left(x_{0}\right)$. Hence $x_{0}$ is also a solution of the VVIP on $K$.

( $\beta$ ) Let $T$ be quasimonotone and inn $K \neq \emptyset$. Since inn $K$ is lineally full, there exists $z \in$ inn $K$ such that $\|z\|<R$. Then it is easy to prove that $z \in$ inn $K_{1}$ (see also the proof of Theorem 3.1 in [10]), so inn $K_{1} \neq \emptyset$. Hence, by Theorem 1 , the VVIP on $K_{1}$ has a solution $x_{0}$, which is in fact, as in the previous case, a solution on $K$.

Note that for a pseudomonotone operator $T$, the assumption of the norm compactness of $T y$ may be replaced by that of compactness. Indeed, if the latter is the case, we set

$$
F_{1}(y)=\{x \in K:(T y)(y-x) \subseteq D(x)\}, y \in K
$$


Then obviously

$$
F_{1}(y) \subseteq F(y), \forall y \in K
$$

Hence Lemma 2 gives

$$
\bigcap_{y \in K} F_{1}(y) \subseteq \bigcap_{y \in K} G(y)
$$

An analogous proof to that of Lemma 3 shows that $F_{1}(y)$ is closed for all $y \in K$. Finally, the proof of Theorem 1 goes through if we consider $F_{1}(y)$ instead of $F(y)$.

If the cone $C$ does not depend on $x$ and $T$ is monotone, then the existence of solutions for the VVIP is a trivial consequence of the analogous theorem for the (scalar) variational inequality problem, as the following shows:

ThEOREM 3. Let $T: K \rightarrow 2^{L(X, Y)} \backslash\{\emptyset\}$ be a monotone, upper hemicontinuous operator with compact values and let $C$ be a cone with nonempty interior in $Y$. Suppose that $K$ is compact or that $X$ is reflexive and $T$ satisfies the coercivity condition of Theorem 2. Then the VVIP

$$
\forall y \in K, \exists A \in T x \text { such that } A(y-x) \notin-\operatorname{int} C
$$

has a solution $x$ on $K$.

Proof: Choose $f \in C^{*} \backslash\{0\}$. Then the operator $f \circ T: K \rightarrow 2^{X^{*}} \backslash\{\emptyset\}$ is obviously monotone, upper hemicontinuous with $w^{*}$-compact values, so there exists a solution $x \in K$ of the variational inequality

$$
\forall y \in K, \exists u \in(f \circ T)(x):(u, y-x) \geqslant 0
$$

(see, for instance, [16]). Obviously, $u=f \circ A$ for some $A \in T x$ and this according to relation (3) shows that $A(y-x) \notin-\operatorname{int} C$, that is, $x$ is also a solution for the VVIP.

REMARK 1 . In the case $Y=\mathbf{R}$, the set of solutions for the (scalar) V.I.P. of the pseudomonotone operator is known to be convex. This does not hold for the VVIP even if the operator $T$ is constant, as the following example shows: Let $X=Y=\mathbf{R}^{2}$, $C(x)=C=\mathbf{R}_{+}^{2}, K=\left\{x \in \mathbf{R}^{2}:\|x\|_{2} \leqslant 1\right\}$ and $T x$ be the identity operator for all $x \in K$. Then $x_{1}=(0,-1)$ and $x_{2}=(-1,0)$ are solutions for the VVIP while all convex combinations of them are not.

REMARK 2. The set $F(y)$ defined by relation (5) is not compact under the assumptions of Theorem 3, as it is asserted to be in the proof of Theorem 2.1 in $[3,2,14]$ (where it is denoted by $\left.F_{2}(y)\right)$. Here is a counterexample: Let $X=Y=\ell_{2}$ and let $B$ be the closed unit ball. Let $\left(e_{n}\right)_{n \in \mathbb{N}}$ be the canonical basis of $\ell_{2}$ and $K=e_{1}+B$. For each 
$x \in K$, let $C(x)=C$, where $C$ is the cone $\bigcup_{\lambda \geqslant 0} \lambda\left(e_{1}+(1 / 4) B\right)$. Note that int $C \neq \emptyset$. For any $y, z$ in $B$ the scalar product $\left\langle e_{1}+y / 4, e_{1}+z / 4\right\rangle$ is positive; it follows that the scalar product of any two elements of $C$ is nonnegative. Hence, $C \subseteq C^{*}$, so in particular int $C^{*} \neq \emptyset$. (This was an additional assumption in [3, Theorem 2.1]). Finally, let $T: K \rightarrow 2^{L\left(\ell_{2}, \ell_{2}\right)}$ be such that $T x$ is the identity operator on $\ell_{2}$ for each $x \in K$. Then $T$ is of course single-valued and monotone. One may immediately check that $F(0)=K \backslash \operatorname{int} C$. It follows that for all $n>1$ we have $e_{1}+e_{n} \in F(0)$ (indeed, otherwise we would have $e_{1}+e_{n}=\lambda\left(e_{1}+z / 4\right)$ for some $z \in B$; this is impossible, since the norm of $(1-\lambda) e_{1}+e_{n}$ is easily seen to be greater than $\left.\lambda / 4\right)$. However, $e_{1}$ is the weak limit of $e_{1}+e_{n}$; on the other hand, since $e_{1} \in \operatorname{int} C$, we have $e_{1} \notin F(0)$, that is, $F(0)$ is not weakly closed.

\section{REFERENCES}

[1] J.M. Borwein and A.S. Lewis, 'Partially finite convex programming, Part I: Quasi relative interiors and duality theory', Math. Programming 57 (1992), 15-48.

[2] G.-Y. Chen, 'Existence of solutions for a vector variational inequality: An extension of the Hartman-Stampacchia theorem', J. Optim Theory Appl. 74 (1992), 445-456.

[3] G.-Y. Chen and X.-Q. Yang, 'The vector complementarity problem and its equivalences with the weak minimal element in ordered spaces', J. Math. Anal. Appl. 153 (1990), 136-158.

[4] G.-Y. Chen and B.D. Craven, 'A vector variational inequality and optimization over an efficient set', $Z$. Oper. Res. 3 (1990), 1-12.

[5] J. Diestel and J.J. Uhl, Vector measures (American Mathematical Society, Providence, R.I., 1970).

[6] N. Dunford and J.T. Schwartz, Linear operators Part I: General theory (Wiley, New York, 1988).

[7] K. Fan, 'A generalization of Tychonov's fixed-point theorem', Math. Annal. 142 (1961), 305-310.

[8] F. Giannessi, 'Theorems of alternative, quadratic programms and complementarity problems', in Variational inequalities and complementarity problems, (R.W. Cottle, F. Giannessi and J.L. Lions, Editors) (Wiley, New York, 1980), pp. 151-186.

[9] N. Hadjisavvas, D. Kravvaritis, G. Pantelidis and I. Polyrakis, 'Nonlinear monotone operators with values in $L(X, Y)$ ', J. Math. Anal. Appl. 140 (1989), 83-94.

[10] N. Hadjisavvas and S. Schaible, 'Quasimonotone variational inequalities in Banach spaces', J. Optim. Theory Appl. 80 (1996), 95-111.

[11] G. Jameson, Ordered linear spaces, Lecture Notes in Mathematics 141 (Springer-Verlag, Berlin, Heidelberg, New York, 1970).

[12] S. Karamardian and S. Schaible, 'Seven kinds of monotone maps', J. Optim. Theory Appl. 66 (1990), 37-46. 
[13] D. Kinderlehrer and G. Stampacchia, An introduction to variational inequalities and their applications (Academic Press, New York, 1980).

[14] G.M. Lee, D.S. Kim, B.S Lee and S.J. Cho, 'Generalized vector variational inequalities and fuzzy extensions', Appl. Math. Lett. 6 (1993), 47-51.

[15] D.T. Luc, 'Characterisations of quasiconvex functions', Bull. Austral. Math. Soc. 48 (1993), 393-406.

[16] M.H. Shi and K.K. Tan, 'Browder-Stampacchia variational inequalities for multivalued monotone operators', J. Math. Anal. Appl. 134 (1988), 431-440.

[17] X.Q. Yang, 'Vector complementarity and minimal element problems', J. Optim. Theory Appl. 77 (1993), 483-495.

\author{
Department of Mathematics \\ University of the Aegean \\ 83200 Karlovassi \\ Samos \\ Greece \\ e-mail: arisd@kerkis.math.aegean.gr \\ nhad@kerkis.math.aegean.gr
}

